\title{
DISCURSOS DE LA FORMACIÓN INVESTIGATIVA DEL CAPITAL INTELECTUAL EN CIENCIAS DE LA SALUD
}

\section{SPEECHES OF THE RESEARCH TRAINING OF INTELLECTUAL CAPITAL IN HEALTH SCIENCES}

\author{
José Marcos Bustos Aguayo*, Sofía López Nava Tapia*, Cruz García Lirios** \\ Universidad Nacional Autónoma de México*, Universidad Autónoma Metropolitana**
}

Correspondencia: bundesnanza@aol.com

\section{RESUMEN}

El objetivo del estudio fue explorar, interpretar y develar los significados en torno a discursos relativos a categorías de transferencia del conocimiento sobre salud en el proceso investigativo. A fin de poder estructurar el sentido de la comunicación entre los interlocutores y discutir las diferencias y las similitudes entre las partes interesadas, considerando la dinámica académica y sus efectos biomédicos, psicológicos y financieros en los Profesores de Tiempo Completo. Se realizó un estudio documental, transversal, exploratorio y comprensivo con una selección intencional de siete informantes claves, Profesores de Tiempo Completo (PTC's) de universidades públicas del centro de México en el área de la salud pública. Se encontró una línea discursiva sobre las disposiciones o habitus profesionales que los informantes expresan ante cuestionamientos sobre su mundo cotidiano académico y profesional. Futuras líneas de investigación en torno a la problemática advertirán las diferencias y similitudes con los hallazgos encontrados y en relación al estado del arte.

Palabras clave: Salud, conocimiento, investigación, docencia, institución. 


\section{ABSTRACT}

The objective of the study was to explore, interpret and unveil the meanings around reluctant speeches to categories of health knowledge transfer in the research process. In order to be able to structure the sense of communication between the interlocutors and discuss the differences and similarities between the stakeholders, considering the academic dynamics and their biomedical, psychological and financial effects on Full-Time Teachers. A documentary, cross-sectional, exploratory and comprehensive study was carried out with an intentional selection of seven key informants, Full Time Professors (PTC's) from public universities in central Mexico in the area of public health. A discursive line was found about the professional dispositions or habitus that the informants express when faced with questions about their daily academic and professional world. Future lines of research around the problem will notice the differences and similarities with the findings found and in relation to the state of the art.

Key words: Health, knowledge, research, teaching, institution.

\section{INTRODUCCIÓN}

En el marco de sociedad de la información y el conocimiento, los investigadores gestionan sus productos; artículos, capítulos o libros para hacer frente a un concurso de méritos y ser reconocidos con becas o financiamiento para sus proyectos (García, 2019). Esto es así porque el concurso de méritos supone una construcción colaborativa, pero ante la falta de recursos, tecnologías, redes y organizaciones, los investigadores asumen individualmente esta labor, atribuyéndola como una extensión de su vocación por el conocimiento y la transferencia de experiencias, saberes y habilidades para con sus alumnos. Por consiguiente, la relación entre investigación y docencia es representada como un compromiso social, académico y laboral, aún y cuando las instituciones designen cada vez menos recursos e incrementen las obligaciones de su profesorado.

Este escenario de desequilibrio entre las demandas institucionales y los recursos profesionales e investigativos es idóneo para la delincuencia quienes engañan a los investigadores al publicar sus artículos en revistas que no cuentan con ningún 
criterio de calidad y cada día cobran un mayor monto por edición de los artículos - libros (García y Carreón, 2018). Mientras tanto, las demandas académicas se intensifican en cuanto a la calidad de procesos y productos con un financiamiento cada vez más reducido. Se trata de un contexto en el que el profesor investigador no sólo recibe las presiones institucionales de su labor, sino además, es vulnerable en cuanto a la difusión de su producción la cual debe costear desde principio a fin, aunque al momento de transferir este conocimiento a sus estudiantes los méritos se desvanecen en el proceso burocrático de reconocimiento.

La dinámica de publicación ha propiciado riesgos y efectos en la salud, principalmente de orden psicológico, aunque cada vez más los casos de afectación biomédica se incrementan en los académicos, principalmente los profesores de tiempo completo (PTC). Es decir que la salud, la calidad de vida y el bienestar subjetivo no se reducen a la figura del investigador. Además, la representación de su labor, recursos, obligaciones y aportes son discontinuos institucionalmente. Tal problemática se exacerba en áreas como la salud que cuenta con discursos distintivos de profesiones especializadas, y actualizadas permanentemente. Es un escenario en el que los investigadores no sólo auto-gestionan, producen y transfieren conocimiento. Además, deben transferir lo que para ellos significa cada tecnicismo a profesionistas sin esa misma formación con el propósito de ser reconocidos en su labor institucional.

Por tanto, es menester llevar a cabo el diseño de espacios institucionales y virtuales en los que los investigadores puedan tener acceso al ranking de universidades, editoriales e investigadores, así como la atención a sus necesidades de salud psicológica, biomédica o financiera. Un proyecto de tal envergadura permitirá la transparencia en la gestión del conocimiento y una base de datos sobre los casos exitosos, áreas de oportunidad y estrategias de gestión, producción y transferencia del conocimiento disminuirá los riesgos de fraudes, plagio y extorsión a los que los investigadores se ven expuestos (García, 2017). Aunado a tal proyecto, la división en cuanto a la gestión, producción y transferencia del conocimiento permitirá establecer indicadores de calidad más comprensivos para el concurso de méritos y el sistema de promociones o compensaciones.

Es así como el objetivo del estudio fue explorar, interpretar y develar los significados en torno a discursos relativos a categorías de transferencia del conocimiento 
sobre salud en el proceso investigativo. A fin de poder estructurar el sentido de la comunicación entre los interlocutores y discutir las diferencias y las similitudes entre las partes interesadas, considerando la dinámica académica y sus efectos biomédicos, psicológicos y financieros en los profesores de tiempo completo.

\section{Teoría de diseño para la salud}

Los marcos teóricos y conceptuales que explican los efectos de las demandas políticas, sociales y educativas con respecto a los recursos institucionales, gremiales y académicos son; 1) teoría de la gestión del conocimiento, 2) teoría del habitus académico y 3) teoría de la salud ocupacional.

Si la relación entre la investigación y el trabajo docente es cultural y políticamente directa, entonces la sociedad, en su afán de crecer requiere del conocimiento de los fenómenos los cuales se pretenden explicar llanamente en un aula para motivar al estudiante a formarse como investigador y garantizar el capital humano que la sociedad requiere para su bienestar y confort (García y Hernández, 2016). En ese tenor, la teoría de la gestión del conocimiento advierte un desbalance entre las demandas sociales de calidad científica y tecnológica respecto a los recursos institucionales. Debido a que el diseño para la salud centra su atención en la prevención de accidentes como de enfermedades, la gestión del conocimiento alude al equilibrio entre los retos y las oportunidades.

Por consiguiente la investigación como proceso de construcción social es una instrumentación de saberes, experiencias, conocimientos y habilidades. Es decir, son medios para el fin último de preservar una civilización (García, Carreón, Bustos, 2015). La gestión del conocimiento aborda esa instrumentación como un discurso especializado y actualizado, considerando un proceso de transferencia de datos en la formación de talentos, pero sin asumir ese escenario como modos de acción y pensamiento en el aula según lo plantea la teoría del habitus académico.

De esta manera, las disposiciones o habitus son modos de hacer y conocer que se traducen en discursos o mensajes distintivos de profesiones e incluso áreas de investigación como a menudo sucede en ciencias de la salud.

Sin embargo, dese el enfoque de la gestión del conocimiento, las habilidades docentes para la investigación, se circunscriben y reducen a la motivación por 
la formación investigativa. El docente es una especie de conciencia práctica que orienta la decisión de dedicarse a la investigación. La motivación del logro, la autoeficacia computacional y la utilidad percibida serian tres factores que compaginan con estilos de vida estudiantil dedicados a la revisión documental y la prueba empírica de modelos (Villegas, Sánchez, Espinoza, García y Quintero, 2019).

En cambio, la teoría de las disposiciones o habitus ubican a la instrumentación del conocimiento en el proceso formativo de talentos. La redacción de textos científicos a partir de las preguntas ¿qué?, ¿cómo?, ¿por qué? pre suponen un profesionista centrado en una línea de investigación, buscando responder a las interrogantes a partir de una revisión sistemática de la literatura, una técnica de procesamiento de información y una motivación hacia las relaciones espurias entre pares (Aldana, Sánchez, Espinoza, Velez, Bustos, Molina, Sandoval y García, 2019). En tal sentido, la teoría de la gestión del conocimiento atribuye al desbalance entre demandas del entorno y recursos institucionales la problemática de la instrumentación de saberes, experiencias y capacidades, pero el enfoque del habitus académico advierte más bien una formación instrumental e intergeneracional sin aludir a sus efectos como la salud ocupacional.

El paradigma de la gestión del conocimiento exacerba el qué de una temática o problemática sugiriendo información disponible, accesible y susceptible de ser estructurada y comparada, aunque reconoce en los datos la particularidad de la investigación (García, Ambrosio, Elizarrarás, Sánchez, Aguilar y García, 2019). El cómo supone una serie de datos ordenados según sus propiedades internas y su relevancia o utilidad externa (García, Quintero, Carreón, Rincón, Martínez, Sánchez y Fierro, 2019). El por qué es una instancia provisional de responder a las dos cuestiones anteriores siempre que otra teoría u ordenamiento de información no contravenga o especifique más eficientemente las respuestas encontradas en una revisión de la literatura (Sánchez, García, García, Juárez, Molina, Amemiya y Martínez, 2019). Las tres preguntas, en el marco de la economía del conocimiento y la sociedad de la información son a menudo dependientes del acceso a los datos, los repositorios y los resúmenes que los usuarios, dependiendo de sus recursos puedan llegar a consultar (Hernández, Carreón, Sánchez, García, Aldana, Martínez, Molina, Juárez y Quintero, 2019). 
En contraposición, la perspectiva del habitus académico propone interpretar los datos con la finalidad de establecer pautas reveladoras del sentido de ciencia y tecnología., pero sin relacionar este horizonte de significados con la salud del investigador. Mientras que la gestión del conocimiento observa la instrumentación de experiencias, habilidades y saberes, el enfoque del habitus académico desmitifica esa formación investigativa en ethos (ética), aesthesis (estética), hexis (aprehensión) y eídos (lógica), aunque sin advertir sus efectos en la salud ocupacional, calidad de vida y bienestar subjetivo.

La teoría de la gestión del conocimiento plantea a la formación investigativa y la adquisición del conocimiento como una praxis que define la elaboración de textos, considerando a la estructura del artículo científico; introducción (para qué, qué hay, qué falta, qué se espera obtener), método (cómo y por qué de esa manera), resultados (qué se encontró), discusión (cuál es su aporte, qué diferencias o similitudes encontró respecto a la literatura revisada), conclusión (se cumplieron los objetivos, cómo, qué falta por hacer).

En contraste, la teoría del habitus académica pretende develar el significado de esa instrumentación al relacionarse con la formación investigativa, pero no como otro instrumento sino como proceso ético, estético, lógico y aprehensivo. Para la gestión del conocimiento la traducción y recodificación de datos es la esencia de la formación investigativa. En el enfoque del habitus académico la instrumentación y recodificación de datos es sólo una instancia de comprensión la cual se extiende hasta la formación distintiva del estudiante con respecto a otros grupos a los que pertenece o desea adscribirse.

Ambas teorías, la gestión del conocimiento y el habitus académico, soslayan los efectos de tal proceso de instrumentación del conocimiento y formación de talentos en los profesores de tiempo completo. Explican debidamente el proceso instrumental y formativo, pero sin considerar el diseño para la salud de los docentes investigadores.

La perspectiva de la salud ocupacional advierte que la prevención de riesgos de accidentes o enfermedades no sólo es inherente a políticas institucionales ante contingencias del entorno. Además, es consustancial a la instrumentación del conocimiento y la formación investigativa porque supone un diseño integral ante el desbalance entre demandas externas y recursos internos a las universidades. 
La mirada de la gestión del conocimiento radica en la investigación como un medio para explorar dudas, construyendo el lenguaje de los textos científicos para la indagación, procesamiento y estructuración de información (Sánchez, Martínez y García, 2019). La óptica del habitus académico consiste en una ética formativa a partir de transferir códigos de saberes, una estética de los datos, una lógica de los razonamientos y una expresividad del conocimiento.

Debido a que internet provee de información gratuita, rápida y fácil a la formación investigativa ésta es asumida como la producción de textos; prontuarios, manuales, recopilaciones, antologías o informes. Se atribuye al investigador el poder de generar literatura exhibida en los estantes de las bibliotecas, cubículos o eventos (Aguilar, Pérez, Pérez, Moralesy García, 2018), aunque detrás de esta producción del conocimiento, la formación de habitus investigativo legitima esa asimetría entre demandas de productos, productividad científica y transferencia de tecnología.

Sin embargo, en las relaciones colaborativas, el cooperativismo, la solidaridad, la confianza, la empatía y el compromiso son fundamentales para lograr el trabajo en equipo (Fierro, López y García, 2018). En el caso de la inteligencia emocional el registro sistemático de solicitudes, asistencias y resultados con base en la cooperación como es el caso de la huella digital que registra la puntualidad y los correos electrónicos que registran las solicitudes y propuestas. Se trata de habitus académicos los cuales se ajustan a la instrumentación del conocimiento, así como al deterioro de la calidad de vida y el bienestar subjetivo de profesores investigadores.

La investigación centrada en el conocimiento del objeto de estudio, asume que un aspecto importante es conocer y ser creativo para poder establecer reflexiones críticas entre pares (Sánchez, Villegas, Sánchez, Espinoza y García, 2018). La investigación inicia con conocimientos previos, vivencias, experiencias cotidianas, que permiten construir y reconstruir en un tiempo y espacio determinado una realidad crítica, pero esta no puede ser desligada del entorno laboral y ocupacional.

La investigación contribuye al desarrollo y modernización del país, hay que impulsar más la investigación en el país, no sólo como actividad nacional estratégica, sino también como método de aprendizaje, apropiación, generación y aplicación de conocimientos (García, Martínez y Sánchez, 2018). Se encuentra ligada al desarrollo educativo y social, al crecimiento económico, la optimización de recursos y la 
revolución organizacional; implica una desconcentración de actividades y recursos; requiere la concertación de esfuerzos y el impulso a una política de masificación de la ciencia y la tecnología según las condiciones específicas de cada región.

\section{Estudios del diseño para la salud}

Los estudios del diseño para la salud centran su interés en la langue como unidad de análisis de los discursos y narrativas de las partes interesadas sobre una temática o problemática común a la gestión, producción y transferencia del conocimiento.

Ricoueur (2006: p. 17) advierte: "Langue es el código o conjunto de códigos" en el que un hablante particular produce parole como un mensaje particular". Por consiguiente, en el ámbito de salud serían; a) símbolos, significados y sentidos biomédicos y biofísicos que coexisten con los biopsicosociales al ser estos últimos parole por su uso excesivo entre médicos, enfermeras, trabajadoras sociales y psicólogos.

Es así como los códigos para tratar los temas de la salud son el biomédico y el biofísico (nacer, crecer, desarrollarse, reproducirse y morir; anafase, profase, telofase, metafase; RNA, DNA; input, output). Esa langue no es del todo asimilado incluso por especialistas; cardiólogos, nutriólogos, urólogos y en consecuencia se difunde en parole, mensajes que aluden al ciclo biomédico-biofísico, pero con un estilo biopsicosocial ("bájale a las grasas y al azúcar", "mente sana en cuerpo sano", "come frutas y verduras", "me duele tantito", "me siento un poquito mal", "no tengo nada", "aguanto todo") asimilable al grueso de profesionales de la salud y usuarios del sistema de atención pública.

De este modo, los mensajes en los temas de salud aspiran a un orden biomédico-biofísico, pero en el proceso: langue $\rightarrow$ parole (locusión, ilocusión, interlocusión) $\rightarrow$ langue...se transforman en retórica, falacias, prejuicios, reduccionismos, otorgando sentido a la comprensión de la negligencia.

Es el caso de la taxonomía de Schliermann propuesta por Pauk (1969) en la que el proceso catedrático investigativo supone fases o instancias desde las que es posible advertir la relación simbiótica entre el ser profesional, docente, investigador y académico en su devenir cotidiano (véase Tabla 1). 
Tabla 1.

Taxonomía de la cátedra del profesor Schliermann

\begin{tabular}{|c|c|c|}
\hline Instancias & Estrategia & Extracto \\
\hline Fundamentación & $\begin{array}{l}\text { Selección } \\
\text { intencional }\end{array}$ & $\begin{array}{l}\text { "el profesor Schliermann tenía "un trato especial con } \\
\text { la coordinación". Podía escoger veinte alumnos nue- } \\
\text { vos para su clase, pero tenía que aceptar otros diez } \\
\text { de la inscripción general" }\end{array}$ \\
\hline Justificación & $\begin{array}{l}\text { Exposición } \\
\text { plenaria }\end{array}$ & $\begin{array}{l}\text { "Schliermann no saludó a los alumnos como era su } \\
\text { costumbre. Se limitó a dar la clase," }\end{array}$ \\
\hline Delimitación & $\begin{array}{l}\text { Motivacional } \\
\text { comunicativa }\end{array}$ & $\begin{array}{l}\text { "si, por ejemplo, elige usted el tema de la conquista } \\
\text { de la Nueva España, puede estar seguro de que no le } \\
\text { va a salir bien, porque no hay posibilidades de cubrir } \\
\text { un asunto tan extenso; iban a ser necesarias docenas } \\
\text { de libros para exponer el tema, no un trabajo de cin- } \\
\text { cuenta páginas. Tenga presente que el título de su } \\
\text { trabajo es una promesa. Algo así como un contrato," }\end{array}$ \\
\hline $\begin{array}{l}\text { Objetivos } \\
\text { Formulación } \\
\text { Hipótesis }\end{array}$ & $\begin{array}{l}\text { Cartográfica } \\
\text { estructural }\end{array}$ & $\begin{array}{l}\text { "Hasta una limitación del asunto, cinéndose única- } \\
\text { mente, por ejemplo a la conquista de Tenochtitlán re- } \\
\text { sultaría todavía demasiado extensa; por tanto, si som- } \\
\text { ete usted el tema a una reducción, como la Batalla de } \\
\text { la Noche Triste, se acercaría más a las proporciones } \\
\text { convenientes, aunque todavía resultaría el asunto de- } \\
\text { masiado extenso; con una cuarta reducción, como la } \\
\text { importancia táctica de los aztecas en la Batalla de la } \\
\text { Noche Triste, se pondría más en el terreno de la real- } \\
\text { idad. Resultaría un tema para el cual podrían reunirse } \\
\text { muchos datos y que cabría perfectamente en un tra- } \\
\text { bajo así, aún tratado con profundidad" }\end{array}$ \\
\hline Resultados & $\begin{array}{l}\text { Analógica } \\
\text { selectiva }\end{array}$ & $\begin{array}{l}\text { "De los cinco restantes decidí hablar detenidamente } \\
\text { con el profesor Schliermann. Manifestó alegría al } \\
\text { verme. En cosa de cinco minutos eliminamos dos" }\end{array}$ \\
\hline Discusión & $\begin{array}{l}\text { Especialización } \\
\text { analógica }\end{array}$ & $\begin{array}{l}\text { "me recomendó que hablase con los profesores es- } \\
\text { pecializados en los campos respectivos" }\end{array}$ \\
\hline Conclusión & $\begin{array}{l}\text { Reducción } \\
\text { sintética }\end{array}$ & $\begin{array}{l}\text { "Si no reúne usted material suficiente de primera } \\
\text { clase, se va a ver en problemas para escribir un tra- } \\
\text { bajo largo" }\end{array}$ \\
\hline
\end{tabular}

Fuente: Pauk (1969) 
La develación del significado de la motivación por investigar es el principio y final de la taxonomía, ya que ésta es cíclica y por ende reproducible en una espiral de tutoría.

Formulación: ¿Cuáles son los significados en torno a los discursos de las partes involucradas en el proceso de investigación, considerando su formación profesional, demandas institucionales y recursos intelectuales?

\section{MÉTODO}

Diseño. Se realizó un estudio documental, exploratorio e interpretativo de los discursos en torno a las partes involucradas en un proceso investigativo del área de la salud pública, considerando su formación profesional, demandas institucionales y recursos intelectuales.

\section{Muestra.}

Datos crudos $=7$ Profesores de Tiempo Completo (PTC)

Datos objetivos $=$ Diseño para la salud a altos y bajos desempeños con riesgos a su salud biomédica, psicológica y financiera

Datos pre-procesados $=$ Redes neuronales para indagar las variables que determinan la salud biomédica, psicológica y financiera

Datos transformados = Capa de salida, estableciendo los efectos

Datos interpretados = Diseño arquitectónico determinado por factores socioeconómicos, socioeducativos, sociodemográficos y socioculturales.

Instrumento. Se elaboró un cuestionario para entrevista a profundidad, considerando la formación profesional (¿Qué significa para usted ser profesionista egresado de su institución?), demandas institucionales (¿Cuál es el significado que para usted guardan las actividades que cotidianamente realiza en su cubículo, el aula, laboratorio o cuando realiza trabajo de campo?), así como recursos intelectuales (¿Tiene algún significado la habilidad que más lo distingue a usted al momento de ejercer su labor docente en el aula o fuera de ella en algún evento?). 
Análisis. Se utilizó la técnica de análisis del discurso sociológico del alejamiento y el posicionamiento narrativo del informante con respecto a reverberaciones de la transferencia del conocimiento. A partir de la categoría de epogue de Husserl, horizonte de comprensión de Gadamer y alejamiento del objeto de Ricoeur se construyó una matriz de extractos y se procesó considerando el tridente organizativo de locución (símbolo), ilocución (significado) e interlocución (sentido) propuesto por Habermans.

\section{RESULTADOS}

En el marco del diagnóstico médico, los datos biomédicos y biofísicos no son accesibles al grueso de profesionales de la salud y se degradan o enriquecen en un léxico biopsicosocial.

Locución 1: "La anamnesis es la base fundamental para el diagnóstico de los problemas de salud de nuestros pacientes. Del 50 al $75 \%$ de los diagnósticos se hacen por el interrogatorio."

llocución 1: Anamnesis o interrogatorio como compromiso de conocimiento y solución al padecimiento, enfermedad o efecto de medicación o tratamiento.

Interlocución 1: Certeza cognitiva en médicos y alivio en pacientes.

Otra subcategoría central en el análisis de la formación profesional, las demandas institucionales y los recursos intelectuales en el área de la salud es el que corresponde a la elaboración del examen médico por el grado de exposición del profesionista a las respuestas y preguntas del paciente.

Locución 2: "El examen físico: complementa al interrogatorio, los signos físicos son "marcas" objetivas y verificables de la enfermedad y representan hechos sólidos e indiscutibles".

Ilocución 2: Fundamentación del compromiso de conocimiento y solución...

Interlocución 2: Medicación e ingesta.

El discurso biopsicosocial se valida por el grado de ajuste de sus signos a los datos biomédicos y biofísicos: 
Locución 3. "en la técnica de asociación de síntomas y signos los médicos tratamos de agrupar los síntomas y signos para realizar el ejercicio diagnóstico, para ello construimos determinadas asociaciones: tríadas, tétradas, y sobre todo, síndromes..."

Ilocución 3. Compromiso de un debido proceso de traducción de un hecho biopsicosocial a un sentido biofísico-biomédico

Interlocución 3. Medicación adecuada (pronta recuperación, convalidación, rehabilitación).

Lo que distingue (constituye) al discurso (proposiciones) de la salud respecto a otros sistemas y estructuras es la coexistencia entre las oraciones biopsicosociales con los datos biomédicos-biofísicos. Es una hermenéutica de interpretación del sentido de estar enfermo, saludable, medio enfermo, medio saludable, medio bien, medio mal, un poco mal o un poco bien:

Locución 4. "Un síndrome es un conjunto de síntomas y signos que de modo frecuente se encuentran asociados, que se relacionan entre sí por medio de una particularidad anatómica, fisiológica o bioquímica, pero que pueden corresponder a etiologías diferentes. No necesariamente identifica la causa precisa de una enfermedad, pero disminuye el número de posibilidades, y a menudo, sugiere la necesidad de practicar ciertos estudios especiales clínicos y de laboratorio. Los trastornos de cada sistema u órgano en el ser humano pueden reducirse a un número relativamente pequeño de síndromes."

Ilocución 4. Compromiso de ajustar los padecimientos, malestares, síntomas a una taxonomía biofísica-biomédica

Interlocución 4. Aporte ideal de nuevos síntomas y signos a los síndromes

\section{DISCUSIÓN}

El aporte del presente trabajo al estado del significado es el análisis discursivo y el develamiento de significados en torno a la transferencia del conocimiento del proceso investigativo en informantes claves del área de la salud comunitaria, aunque el diseño de la investigación limitó la interpretación a los participantes, 
sugiriendo la extensión del mismo a la codificación de escenarios, pasajes y sujetos receptores de la enseñanza.

En relación con el estado de la cuestión en donde la construcción del significado de la salud va más allá que su simbolismo investigativo, el presente trabajo ha puesto de relieve que en la dimensión comunicativa, las partes interesadas resultan tener diferencias no por sus posicionamientos, sino por su devenir histórico de lo que para ellos significa estar saludable.

Es decir, un posicionamiento discursivo de la investigación para la salud no puede ser sustentado desde la praxis ni siquiera desde su ontología, ya que se trata de la transferencia de formatos de asimilación del conocimiento que a menudo se imponen a la racionalidad o la comprensión de un fenómeno.

El estado del arte al señalar que la forma se ajusta al fondo en cuanto a la diseminación de redes colaborativas en salud sugiere que el proceso investigativo se gesta en el laboratorio, soslayando la construcción de la disciplina o el paradigma en el aula o fuera de ella, en el campo laboral.

El presente trabajo advierte que las demandas institucionales condicionan la prevalencia de la forma sobre el fondo y que los investigadores al no resignificar la importancia de su labor en la instrumentación como la de salvar vidas, generan una separación entre la utilidad que supone el mercado y la vocación que significa la academia.

Por último, los trabajos sobre la búsqueda del sentido de la profesión, el desarrollo de las habilidades y los conocimientos sugiere que las partes involucradas en el proceso investigativo, heredan los recursos personales, pero en el presente trabajo más bien se destaca que prevalece un habitus profesional o una disposición a cooperar que culmina con la atención pública.

Futuras investigaciones en el área especializada de la salud pública develarán significados ya no en torno a la profesión ni al lugar de trabajo sino a las habilidades y conocimientos adquiridos y fundamentalmente las destrezas y capacidades por adquirir ya que se observa una disposición favorable a la sabiduría. 


\section{CONCLUSIÓN}

El objetivo del estudio fue explorar, interpretar y develar los significados en torno a discursos relativos a categorías de transferencia del conocimiento sobre salud en el proceso investigativo, pero el diseño del estudio limitó los resultados a la muestra seleccionada intencionalmente, sugiriendo la extensión del trabajo a otras áreas como las prácticas profesionales y el servicio social, principales escenarios de alianzas estratégicas entre instituciones públicas y organizaciones ante las demandas del mercado local.

A fin de poder estructurar el sentido de la comunicación entre los interlocutores y discutir las diferencias y las similitudes entre las partes interesadas, considerando la dinámica académica y sus efectos biomédicos, psicológicos y financieros en los Profesores de Tiempo Completo el presente trabajo recomienda puntualizar la gestión del conocimiento ante el aumento de su instrumentación, la reducción de la formación investigativa y el incremento de efectos consustanciales a la docencia, investigación y salud ocupacional.

El diseño para la salud de los profesores investigadores supone la gestión de su conocimiento, así como la formación de talentos, pero también la prevención de accidentes y enfermedades inherentes a los riesgos laborales y ocupacionales que los envuelve. Es decir, los significados develados de sus discursos sugieren escenarios de posicionamiento de su discurso para la apropiación de espacios preventivos.

La construcción de un entorno saludable para los profesores investigadores va más allá de incentivos, reconocimientos, ceremonias, investiduras, concesiones o atención médica. El diseño para su salud implica la construcción y la deconstrucción de sus productos ya no a partir de la calidad sino desde una ética, estética, lógica y expresividad de sus disposiciones a la investigación.

En cuanto a la transferencia de conocimiento, los profesores de tiempo completo tienen ante sí el desafió de redireccionar su comunicación, codificar y recodificar sus locuciones, ilocuciones e interlocuciones hacia la creación de un escenario preventivo de riesgos y amenazas a su salud. 


\section{REFERENCIAS}

I Aguilar, J. A., Pérez, G., Pérez, I., Morales, M. L. y García, C. (2018). Gobernanza de las redes de conocimiento: contrastación de un modelo para el estudio de la formación consensuada. Alternativas, 40, 24-50

I Aldana, W. I., Sánchez, A., Espinoza, F., Velez, S. S., Bustos, J. M., Molina, H. D., Sandoval, F. R. y García, C. (2019). Gobernanza de la responsabilidad de salud en una ciudad del centro de México. Nueva Enfermería, 19 (1), 5-17

I Fierro, E., López, S. y García, C. (2018). Confiabilidad y validez de un instrumento que mide el compromiso organizacional en un centro de salud comunitaria. Tlatemoani, 29, 42-68

I García, C. (2017). Gobernanza de la Salud Pública. México: UAEM-UAP Huehuetoca

1 García, C. (2019). Especificación de un modelo para el estudio de la flexibilidad laboral en IES y mipymes formadoras del capital intelectual. Tlatemoani, 30, 67-89

I García, C. y Carreón, J. (2018). Gobernanza universitaria. México: UNAM-ENTS

I García, C. y Hernández, J. (2016). Gobernanza de la vida sociopolítica. Sociológica, 3 (2), 45-34

I García, C., Ambrosio, O., Elizarrarás, G., Sánchez, R., Aguilar, J. A. y García, E. (2019). Contraste de un modelo de gestión educativa. Eureka, 16 (1), 100-115

I García, C., Carreón, J., Bustos, J. M. (2015). Psicología ambiental. México: UNAM-FES Zaragoza

I García, C., Martínez, E. y Sánchez, A. (2018). Estructura factorial exploratoria de las dimensiones institucionales del compromiso laboral en una Institución de educación Superior (IES) del centro de México. Perspectivas, 20 (2), 65-87

I García, C., Quintero, M. I., Carreón, J., Rincón, R. M., Martínez, E., Sánchez, A. y Fierro, E. (2019). Redes formativas inteligentes en una universidad pública del centro de México. Epsys, 12 (1), 1-13

I Hernández, J., Carreón, J., Sánchez, A., García, C., Aldana, W. I., Martínez, E., Molina, H. D., Juárez, M. y Quintero, M. L. (2019). Exploratory conceptual structure of intellectual capital training organizations. Journal of Information Science, 3 (1), 1-4 
I Pauk, W. (1969). The research paper; Time and technique. Journal of Reading, 13, 15-56.

I Ricoeur, P. (2006). Teoría de la interpretación. México: Siglo XXI

1 Sánchez, A., García, C., García, J. J., Juárez, M., Molina, H. D., Amemiya, M. y Martínez, E. (2019). Effects of corporate governance policies on the quality of technological life. International Journal of Innovative Technology and Exploring Engineering, 10 (1), 1-14

I Sánchez, A., Martínez, E. y García, C. (2019). Exploratory factor structure of labor commitment. International Journal of Technology, 9 (1), 1-8

I Sánchez, R., Villegas, E., Sánchez, A., Espinoza, F. y García, C. (2018). Modelo para el estudio de la lucidez organizacional y la responsabilidad social corporativa. Sincronía, 22 (74), 467-483

I Villegas, E., Sánchez, A., Espinoza, F., García, C. y Quintero, M. L. (2019). Estructura factorial exploratoria del capital humano intelectual. Foro educacional, 32, 31-51

Envío a dictamen: 30 de agosto de 2019

Reenvío: 16 de octubre de 2019 Aprobación: 25 de octubre de 2019 